\title{
LETTERS
}

\section{Mental health care and suicide in pregnancy and postpartum}

The study by Dr. Grigoriadis and colleagues ${ }^{1}$ on suicide rates in Ontario during pregnancy and the first year postpartum shows the importance of the use of combined data sources to obtain a more accurate overview of numbers of suicides than might be available from coroner's reports or administrative data alone. Their results are also important because they situate these suicides within the broader framework of suicide among women of reproductive age over the same time period.

One of the most striking findings is that a high proportion of the pregnant and postpartum women who committed suicide had received mental health care within the last year, predominantly within a primary care setting. It would be useful to know more about the type of care provided. What proportion of these women were taking antidepressants when they committed suicide versus obtaining psychotherapy or other nondrug treatments for depression and anxiety disorders? How does this compare with patterns of mental health care provided to the nonperinatal suicide group and the subset of perinatal controls with a psychiatric diagnosis?

\section{Barbara Mintzes PhD}

Senior Lecturer, Charles Perkins Centre and Faculty of Pharmacy, The University of Sydney, Sydney, Australia

- Cite as: CMAJ 2017 December 4;189: E1498. doi: $10.1503 / \mathrm{cmaj} .733429$

\section{Reference}

1. Grigoriadis S, Wilton AS, Kurdyak PA, et al. Perinatal suicide in Ontario, Canada: a 15-year population-based study. CMAJ 2017;189:E1085-92.

Competing interests: None declared. 